\title{
$\phi$ Production in Proton-Nucleus and Indium-Indium Collisions at the CERN SPS
}

\author{
M. Floris*, R. Arnaldi ${ }^{\dagger}$, R. Averbeck**, K. Banicz ${ }^{*}$, , J. Castor ${ }^{\mathbb{I}}$,
} B. Chaurand $\|$, C. Cicalo*, A. Colla ${ }^{\dagger}$, P. Cortese ${ }^{\dagger}$, S. Damjanovic ${ }^{\S}$, A. David ${ }^{\ddagger}$, , A. De Falco*, A. Devaux ${ }^{\mathbb{I l}}$, A. Drees**, L. Ducroux ${ }^{*}$, H. En'yo ${ }^{\S \S}$, A. Ferretti ${ }^{\dagger}$, P. Force ${ }^{\mathbb{I}}$, N. Guettet ${ }^{\ddagger}, \mathbb{I}$, A. Guichard ${ }^{\ddagger}$, H. Gulkanian ${ }^{\text {III }}$, J. Heuser ${ }^{\S \S}$, M. Keil ${ }^{\ddagger \dagger \dagger}$, L. Kluberg ${ }^{\ddagger}, \|$, J. Lozano ${ }^{\dagger \dagger}$, C. Lourenço ${ }^{\ddagger}$, F. Manso ${ }^{\mathbb{I}}$, A. Masoni*, P. Martins ${ }^{\ddagger}, \dagger$, A. Neves ${ }^{\dagger \dagger}$, H. Ohnishi ${ }^{\S}$, C. Oppedisano ${ }^{\dagger}$, P. Parracho ${ }^{\ddagger}$, P. Pillot ${ }^{\ddagger}$, G. Puddu*, E. Radermacher ${ }^{\ddagger}$, P. Ramalhete ${ }^{\ddagger}$, P. Rosinsky ${ }^{\ddagger}$, E. Scomparin ${ }^{\dagger}$, J. Seixas ${ }^{\ddagger}, \dagger$, S. Serci*, R. Shahoyan ${ }^{\ddagger}, \dagger$, P. Sonderegger ${ }^{\dagger \dagger}$, H.J. Specht ${ }^{\S}$, R. Tieulent ${ }^{\ddagger}$, G. Usai*, R. Veenhof ${ }^{\ddagger}, \dagger$ and H.K. Wöhri

\author{
*Univ. di Cagliari and INFN, Cagliari, Italy \\ ${ }^{\dagger}$ Univ. di Torino and INFN, Italy \\ ** SUNY Stony Brook, New York, USA \\ $\stackrel{\doteqdot}{\doteqdot} E R N$, Geneva, Switzerland \\ ${ }^{\S}$ Univ. Heidelberg, Heidelberg, Germany \\ ${ }^{I} L P C$, Univ. Blaise Pascal and CNRS-IN2P3, Clermont-Ferrand, France \\ ${ }$ LLR, Ecole Polytechnique and CNRS-IN2P3, Palaiseau, France \\ ${ }^{\dagger}$ IST-CFTP, Lisbon, Portugal \\ IPN-Lyon, Univ. Claude Bernard Lyon-I and CNRS-IN2P3, Lyon, France \\ ${ }^{\S}$ RIKEN, Wako, Saitama, Japan \\ III YerPhI, Yerevan, Armenia
}

\begin{abstract}
The quality of the dimuon measurements made by NA60, in proton-nucleus and heavyion collisions, is much better than that reached by previous experiments, such as NA38 and NA50. The most important improvement is due to the use of a radiation-tolerant silicon vertex telescope, placed immediately downstream of the target. This allows NA60 to do a high quality measurement of $\phi$ meson yields and $p_{\mathrm{T}}$ distributions.

This paper presents results obtained in $\mathrm{p}-\mathrm{Be}, \mathrm{p}$ - $\mathrm{In}$ and $\mathrm{p}-\mathrm{Pb}$ collisions at $400 \mathrm{GeV}$, from data collected in 2002, and in In-In collisions at $158 \mathrm{AGeV}$, as a function of centrality, from the 2003 running period. In particular, we show that the inverse $m_{\mathrm{T}}$ slope measured in In-In collisions, in the $\phi \rightarrow \mu \mu$ decay channel, increases with the number of nucleons participating in the collisions, rather than following a flat trend as seen in the NA50 data collected in the same decay channel but restricted to high $p_{\mathrm{T}}$ values. We also show that our measurements seem to agree with the values previously measured by NA49, using $\phi \rightarrow$ KK decays, in $\mathrm{Pb}-\mathrm{Pb}$ and other collision systems.
\end{abstract}

Keywords: Heavy ion collisions, proton-nucleus collisions, $\phi$ meson, dimuon PACS: 25.75.-q,25.75.Dw,14.40.Cs

NA60 is a fixed-target experiment devoted to the study of dimuon production in proton-nucleus (p-A) and nucleus-nucleus collisions at the CERN SPS. Its apparatus is composed of 4 main detectors: a muon spectrometer, a zero degree calorimeter (ZDC), a beam tracker and a vertex detector. A detailed description of the apparatus can be found 
in Ref. [1, 2]. Here we only briefly mention the detector concept.

The muon spectrometer is placed after a hadron absorber, which stops most hadrons before they can reach trigger hodoscopes and tracking chambers. The muon spectrometer also provides the main trigger to the experiment, the "dimuon trigger". To make sure that only muons can trigger the experiment, the hadron absorber is complemented by a 1.2-meter-long iron wall, placed before the last trigger station at the end of the muon spectrometer. The absorber represents the main limiting factor in the spectrometer, because of fluctuations of energy loss and multiple scattering which result in a degraded resolution of dimuon mass and of the coordinates of the interaction vertex.

The vertex detector reconstructs all charged tracks before the hadron absorber. In order to identify the muons among these, the tracks reconstructed in the muon spectrometer are extrapolated back to the vertex region and matched to the tracks reconstructed in the vertex detector. This matching is done both in coordinate and in momentum space. Once identified, the muons are refitted using the joint information of the muon spectrometer and of the vertex detector. We shall refer to these tracks as "matched muons". This technique allows to overcome the limitation due to the hadron absorber and thus results in much improved mass resolution and vertexing capability with respect to previous dimuon experiments. The mass resolution goes from around $80 \mathrm{MeV}$ to $23 \mathrm{MeV}$ at the $\phi$, when using the information from the vertex detector. The resolution on the determination of the vertex position is $\sim 20 \mu \mathrm{m}$ in the transverse coordinates and better than $200 \mu \mathrm{m}$ for the longitudinal coordinate. Furthermore, the dipole field in the target region significantly increases the acceptance of low $p_{\mathrm{T}}$ and low mass dimuons (Fig. 1).

In this work, we present results on $\phi$ meson production in heavy ion collisions. These studies are motivated by the fact that the $\phi$ meson carries information about strangeness production [3]. The yield and $p_{\mathrm{T}}$ spectrum of this meson have been studied in $\mathrm{Pb}-\mathrm{Pb}$ collisions at $158 \mathrm{AGeV}$ incident beam energy by the NA49 (in the $\phi \rightarrow$ $K K$ channel [4]) and the NA50 (in the $\phi \rightarrow \mu \mu$ channel [5]) experiments. They both estimated the inverse slope parameter $T$ fitting $p_{\mathrm{T}}$ spectra with an exponential function. The $T$ values found were in strong disagreement, both in what concerns the absolute value and the centrality dependence. NA50 values were significantly lower than NA49 ones and showed no dependence on centrality. NA49 values, on the other hand, were shown to rise as a function of the number of participants. This discrepancy is also known as the " $\phi$ puzzle" [6].

In this paper we report new measurements in the muon channel done by the NA60 collaboration in p-A collisions (400 GeV incident beam energy) and In-In collisions (158 AGeV incident beam energy), which can help understanding the $\phi$ puzzle. NA60, in fact, can measure the $\phi \rightarrow \mu \mu$ channel with very good $p_{\mathrm{T}}$ coverage and the new collision system adds further information to the general systematics. NA60 can also have access to the $\phi \rightarrow K K$ channel, by means of charged tracks reconstructed in the vertex telescope. In this work we only report on results in the dimuon channel, as the $\phi \rightarrow K K$ studies are still at a preliminary level. At present, the measurement was shown to be feasible with Monte Carlo simulations, but the background subtraction in the real data still needs to be tuned.

To extract the information on $\phi$ production we used spectra of matched muons. These are affected by two sources of background: the combinatorial background and the fake matches. The former is the contribution of uncorrelated muon pairs coming from the 
Table 1. Centrality bins.

\begin{tabular}{cccc}
\hline bins & $\begin{array}{c}\mathrm{d} N_{c h} / \mathrm{d} \eta \\
\text { range }\end{array}$ & $\left\langle\mathrm{d} N_{c h} / \mathrm{d} \eta\right\rangle$ & $\left\langle N_{\text {part }}\right\rangle$ \\
\hline 1 & $4-28$ & 16 & $\sim 20$ \\
2 & $28-92$ & 70 & 91 \\
3 & $92-160$ & 145 & 161 \\
4 & $>160$ & 200 & 197 \\
\hline
\end{tabular}

decay of pions and kaons. The latter comes from the fact that the matching procedure can fail and a muon can be associated to a wrong track in the vertex telescope. When this happens, the kinematics of the matched muon is highly degraded. The combinatorial background is subtracted with an event mixing technique [7]. The fake matches contribution is estimated via simulation, reconstructing a Monte Carlo dimuon on top of a real event ("overlay Monte Carlo"). This contribution can be alternatively estimated with an event mixing tecnique [7]. The two methods agree within $5 \%$.

In order to extract particle yields this clean sample is fitted with the expected sources. These are (two body and dalitz) leptonic decays of low mass mesons and an underlying continuum mostly due to the simultaneous semi-muonic decay of two D mesons.

The decays included in the expected sources are: $\eta \rightarrow \mu \mu, \omega \rightarrow \mu \mu, \phi \rightarrow \mu \mu$, $\rho \rightarrow \mu \mu, \omega \rightarrow \mu \mu \pi^{0}, \eta \rightarrow \mu \mu \gamma, \eta^{\prime} \rightarrow \mu \mu \gamma$.

The p-A data were collected in 2002 with a $400 \mathrm{GeV}$ proton beam. Six targets were installed in the experiment: In, $\mathrm{Pb}$ and four Be disks, $2 \mathrm{~mm}$ thick each. The statistics in the sample is rather low $(\sim 15000$ events after background subtraction and event selection). It was collected in only four days of data taking. The fit of the mass spectra with the expected sources allowed to extract the $\phi / \omega$ cross section ratio. The results we got are $0.062 \pm 0.004$ for $\mathrm{p}$-Be, $0.083 \pm 0.007$ for $\mathrm{p}$-In and $0.081 \pm 0.006$ for $\mathrm{p}-\mathrm{Pb}$. The quoted errors are purely statistical. The p-Be result, in particular, is in agreement with a previous measurement by HELIOS-1 [8]. As can be seen from these results, $\phi$ production is already enhanced between $\mathrm{p}-\mathrm{Be}$ and $\mathrm{p}-\mathrm{Pb}$ collisions.

The indium-indium data were collected with a $158 \mathrm{AGeV}$ ion beam in a 5-week-long run in 2003. In this paper we present results based on $50 \%$ of the very high collected statistics ( $\sim 570000$ events after background subtraction). The data were divided in four centrality bins as summarized in Table 1 . The bins were selected using the number of charged tracks reconstructed in the vertex telescope. The corresponding number of participants was estimated with a Glauber fit to the ZDC energy spectrum. We studied the $\phi / \omega$ cross section ratio and the $p_{\mathrm{T}}$ spectrum of the $\phi$.

To extract the cross section ratios, the mass spectra were fitted independently in the four centrality bins. The parameters allowed to vary were $\eta / \omega, \rho / \omega, \phi / \omega$ and the normalization of the continuum. Fitted spectra were arbitrarily normalized to the $\omega$ peak, in order to be compared to the data.

Figure 1 shows the acceptance of the experiment in the low mass and low $p_{\mathrm{T}}$ region. In order to check if this complex acceptance is under control and if, in general, the apparatus is well understood, the first step in the analysis was a careful study of the most peripheral bin. This was analyzed in three $p_{\mathrm{T}}$ windows. The $\eta / \omega$ and the $\phi / \omega$ ratios were found to be nearly $p_{\mathrm{T}}$ independent. The value of the $\eta / \omega$ ratio is in good agreement 

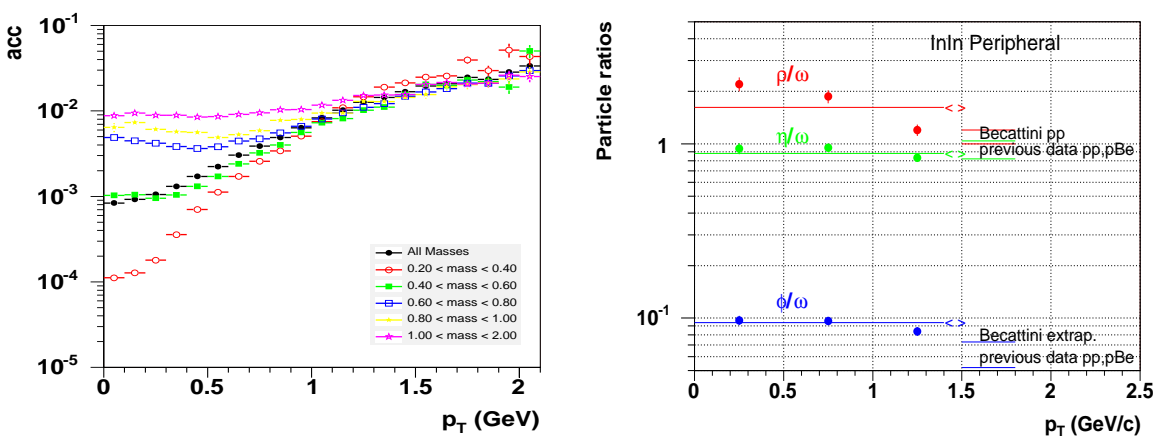

Figure 1. Dimuon acceptance as a function of $p_{\mathrm{T}}$ in several mass windows (left) and particle ratios in the most peripheral bin as a function of $p_{\mathrm{T}}$ (right).

with previous pp and $\mathrm{p}-\mathrm{Be}$ measurements [9]. The $\phi / \omega$ ratio is higher than in $\mathrm{pp}$, as expected in nucleus-nucleus collisions. In general the peripheral bin is well described in terms of expected sources, indicating that the acceptances in the low mass and low $p_{\mathrm{T}}$ region are under control. The $\rho / \omega$ ratio, however, shows that there are "too many" $\rho$ mesons at low $p_{\mathrm{T}}$. This "excess" can be interpreted as the effect of pion annihilation in nuclear collisions. Peripheral In-In collisions, thus, are similar to $\mathrm{C}-\mathrm{C}$ or $\mathrm{O}-\mathrm{O}$ and not just pp-like. It can be shown that at high $p_{\mathrm{T}}(\gtrsim 1 \mathrm{GeV})$ this contribution becomes negligible, as one recovers the expected $\rho / \omega$ ratio of 1.2. The effect of pion annihilation becomes dramatic in more central collisions, but NA60 can still extract a robust $\omega$ yield, thanks to its excellent mass resolution. For a detailed discussion of the modification of the $\rho$ meson in In-In collisions see Ref. [10]. To avoid this problematic region we decided to restrict our analysis of the particle ratios to the region $\mathrm{p}_{\mathrm{T}}>1 \mathrm{GeV}$, until the continuum below the $\omega$ is fully under control.

The $\phi / \omega$ ratio was studied as a function of centrality and compared to previous measurements. We observe a factor 2 increase from peripheral to central collisions. Figure 2 shows the ratio compared to the $\phi /(\rho+\omega)$ ratio measured by NA50 in Pb$\mathrm{Pb}$ collisions [5]. The NA50 points have a common $m_{\mathrm{T}}$ cut $\left(m_{\mathrm{T}} \geq 1.5 \mathrm{GeV}\right)$, so we corrected them to a common $p_{\mathrm{T}}$ range, assuming the $T$ parameter measured by NA50 itself $(228 \mathrm{MeV})$. The $\phi /(\rho+\omega)$ was finally converted to $\phi / \omega$ assuming $\sigma_{\rho}=1.2 \sigma_{\omega}$. The trend as a function of centrality is the same in the two experiments, albeit the absolute value of NA60 points is lower. A direct comparison, however, is impossible due to the contribution of pion annihilation discussed above: this must be even higher in $\mathrm{Pb}-\mathrm{Pb}$ collisions and NA50 cannot isolate it. Figure 2 shows the comparison to the ratio $\phi / \pi$ measured by the NA49 experiment. The dependence on centrality is the same also in this case, indicating that the $\omega / \pi$ ratio is constant, as expected in statistical models. If we use the value $\omega / \pi$ suggested by such models $(0.07-0.08)$ we find that the $\phi$ yield in NA60 is higher than that measured by NA49.

To study the $p_{\mathrm{T}}$ spectrum of the $\phi$ we selected events in the mass window $0.98<$ $M_{\mu \mu}<1.06$. The $p_{\mathrm{T}}$ spectrum of the continuum below the $\phi$ was estimated and subtracted selecting events in two side mass windows $\left(0.88<M_{\mu \mu}<0.92\right.$ and $1.12<$ 

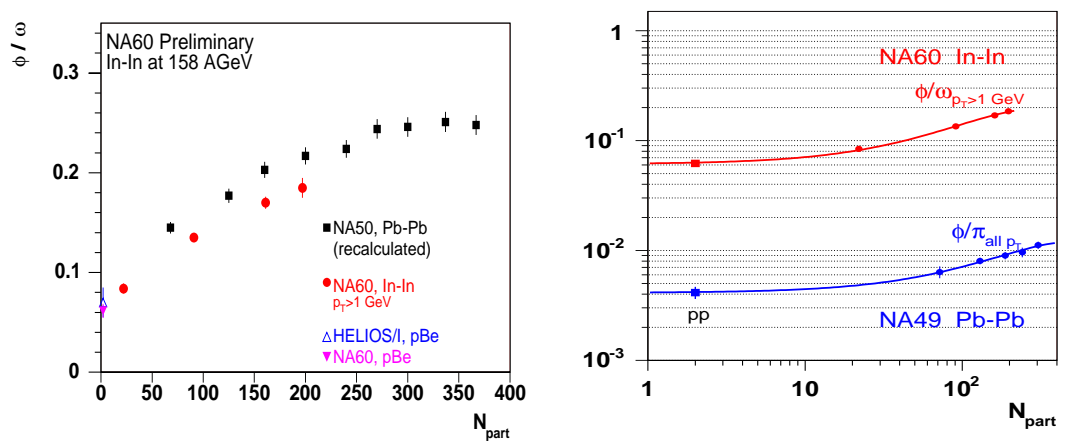

Figure 2. $\phi / \omega$ cross section ratio as a function of centrality compared to the $\phi /(\rho+\omega)$ of NA50 (left) and to the $\phi / \pi$ ratio of NA49 (right). See text for a discussion on how NA50 points were corrected. Statistical errors only.
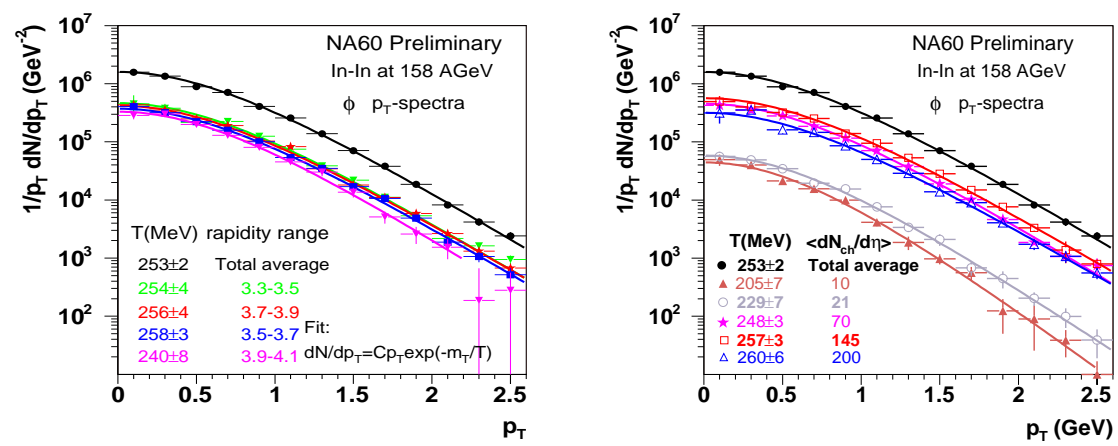

Figure 3. $\phi$ transverse momentum distribution in indium-indium collisions; as a function of rapidity (left) and centrality (right). Statistical errors only.

$\left.M_{\mu \mu}<1.16\right)$. The $p_{\mathrm{T}}$ spectrum was then corrected for acceptance, estimated by Monte Carlo, as a 2-dimensional matrix in $p_{\mathrm{T}}$ and rapidity. The resulting distributions were studied as a function of rapidity and as a function of centrality. The study as a function of centrality was done in five bins, splitting the most peripheral one in 2 sub-bins. The spectra were fitted with an exponential function:

$$
\frac{1}{\mathrm{p}_{\mathrm{T}}} \frac{\mathrm{d} N}{\mathrm{dp}_{\mathrm{T}}}=C e^{-\mathrm{m}_{\mathrm{T}} / T}
$$

The results of the fit are reported in Fig. 3.

No dependence of $T$ on rapidity is observed, while a clear increase with centrality is seen. The average value of the $T$ slope parameter is $T=(253 \pm 2) \mathrm{MeV}$. Changing the fit range to the NA49 $\left(p_{\mathrm{T}}<1.5 \mathrm{GeV}\right)$ or NA50 $\left(m_{\mathrm{T}}>1.5 \mathrm{GeV}\right)$ windows produces small variations on the result: $T=(260 \pm 5) \mathrm{MeV}$ and $T=(244 \pm 5) \mathrm{MeV}$, respectively. 

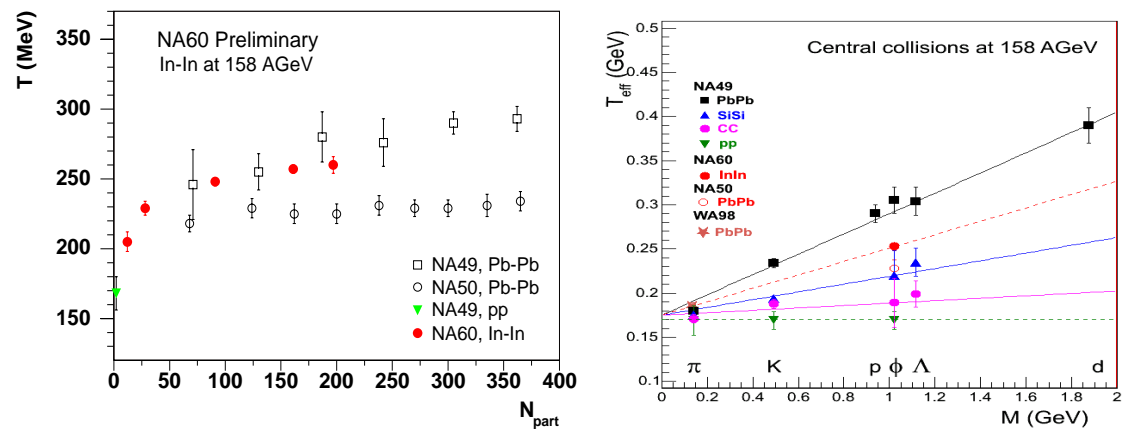

Figure 4. $T$ slope parameter as a function of the number of participants (left) and as a function of particle mass for several collision systems (right). Statistical errors only.

Our results are in agreement with NA49. This can also be seen in Fig. 4, which shows the centrality dependence of the T slope parameter, compared to the analogous result obtained by NA49 and NA50. Our average value, moreover, fits very well in the NA49 systematics [11], the In-In point lying in between the $\mathrm{Pb}-\mathrm{Pb}$ and the $\mathrm{Si}$-Si points (Fig. 4). $\mathrm{NA} 50 \mathrm{~Pb}-\mathrm{Pb}$ result, on the other hand, is quite close to NA49 Si-Si.

We can therefore conclude that the disagreement between NA50 and NA49 was not due to the different decay channels probed.

In this paper NA60 results on $\phi$ production in p-A collisions at $400 \mathrm{GeV}$ incident beam energy and In-In collisions at $158 \mathrm{GeV}$ incident beam energy were presented. The $\phi / \omega$ cross section ratio measured by NA60 in p-A collisions is in agreement with previous measurements. The trend with centrality in nuclear collisions agrees with previous measurements of the $\phi / \pi$ ratio by NA49 (assuming a constant $\omega / \pi$ ) and of the $\phi /(\rho+\omega)$ ratio by NA50 (properly corrected). The absolute yield, however, seems to lie in between these two. The $p_{\mathrm{T}}$ spectrum of the $\phi$ was fitted with an exponential function and studied as a function of centrality and rapidity, giving results which agree with the previous measurement of the NA49 experiment.

\section{REFERENCES}

1. A. Baldit, et al., CERN-SPSC-2000-010 (2000).

2. M. Floris, et al., IEEE Trans. Nucl. Sci. 51 (2004).

3. J. Rafelski, and B. Muller, Phys. Rev. Lett. 48, 1066 (1982).

4. S. V. Afanasev, et al., Phys. Lett. B 491, 59-66 (2000).

5. B. Alessandro, et al., Phys. Lett. B 555, 147-155 (2003).

6. E. V. Shuryak, Nucl. Phys. A 661, 119-129 (1999), hep-ph/9906443.

7. R. Shahoyan, et al., "Charm and intermediate mass dimuons in In+In collisions," in Proc. Quark Matter 2005, Budapest, 2005, in print.

8. R. J. Veenhof, Ph.D. thesis, Universiteit van Amsterdam (1993), rx-1433 (Amsterdam).

9. G. Agakishiev, et al., Eur. Phys. J. C 4, 231-247 (1998).

10. S. Damjanovic, et al., "Low mass dimuon production in Indium-Indium collisions at $158 \mathrm{AGeV}$ (NA60)," in Proc. Quark Matter 2005, Budapest, 2005, in print.

11. I. Kraus, et al., J. Phys. G 30, S583-S588 (2004), nucl-ex/ 0306022. 